OPEN ACCESS

Edited by:

Eugene Dempsey,

University College Cork, Ireland

Reviewed by:

Kazumichi Fujioka, Kobe University, Japan

Brian Henry Walsh,

Cork University Maternity

Hospital, Ireland

${ }^{*}$ Correspondence:

Shuping Han

shupinghan@njmu.edu.cn

tThese authors have contributed equally to this work

Specialty section:

This article was submitted to Neonatology

a section of the journal

Frontiers in Pediatrics

Received: 31 March 2020 Accepted: 10 August 2020 Published: 20 October 2020

Citation:

Xu L, Chen W, Wang X, Yu Z and Han S (2020) Comparative Lipidomic Analyses Reveal Different Protections in Preterm and Term Breast Milk for Infants. Front. Pediatr. 8:590 doi: 10.3389/fped.2020.00590

\section{Comparative Lipidomic Analyses Reveal Different Protections in Preterm and Term Breast Milk for Infants}

\author{
Liping $\mathrm{Xu}{ }^{1+}$, Wenjuan Chen ${ }^{2 t}$, Xingyun Wang ${ }^{2}$, Zhangbin $\mathrm{Yu}^{2}$ and Shuping Han ${ }^{2 *}$ \\ ${ }^{1}$ Department of Pediatrics, Zhangzhou Affiliated Hospital of Fujian Medical University, Zhangzhou, China, ${ }^{2}$ Department of \\ Pediatrics, Women's Hospital of Nanjing Medical University, Nanjing Maternity and Child Health Care Hospital, Nanjing, China
}

\begin{abstract}
Aim: Neonates are notably vulnerable, however they have improved outcomes if they are fed human milk. Human milk lipids constitute the primary constituents of human milk and serve a pivotal role in safeguarding infants from diseases. We assessed the lipid differences between preterm and term human milk and predicted the prospective impacts of these lipids on the development of neonates.
\end{abstract}

Methods and results: We collected colostrum from healthy breast-feeding mothers who had delivered either term or preterm infants. We analyzed the lipid profiles of preterm, as well as term human milk using an LC-MS/MS metabolomics strategy. The orthogonal partial least-squares discriminant analysis score plots revealed remarkable distinction of lipids in preterm and term human milk. In total, 16 subclasses of 235 differential lipids (variable importance in projection $>1, P<0.05$ ) were identified. Notably, phosphatidylethanolamine and phosphatidylcholine were robustly increased in preterm human milk, while diacylglycerol and ceramide were markedly decreased in preterm human milk. Pathway analysis revealed that these dysregulated lipids are closely associated with glycerophospholipid metabolism, sphingolipid metabolism, Reelin signaling in neurons, and LXR/RXR activation.

Conclusion: The results show that the lipids in preterm and term human colostrum vary, which may be critical for neonatal development.

Keywords: colostrum, lipids, LC-MS/MS, neonate, preterm

\section{INTRODUCTION}

Human milk constitutes an optimal supply of nutrition and bioactive components for infants (1). Furthermore, human milk feeding is known to have several important specific protective actions and is recommended for preterm infants (2). Preterm neonates that consume human milk have improved immunity (3), decreased morbidity and mortality associated with necrotizing enterocolitis (4), better neurodevelopmental outcomes, and improved long-term health outcomes $(5,6)$. However, human milk composition is variable during the 1st weeks of life because the nutritional needs of preterm infants are different from those of term infants (7). Previous studies have investigated the constitution of preterm, as well as term human milk, indicating that significant differences are important for infant development (8). 
Lipids constitute the majority of the macronutrient components in human milk and exert a nutritional effect. In addition, human milk lipids also play specialized roles in gastrointestinal function, infant growth, neurodevelopment, and immunity (9). Lipidomics is a powerful analytical approach and can be used to inspect the lipid metabolic reaction of living systems in intricate biological specimens (10). Recently, lipidomics has made significant research advancements on human milk, due to its great advances of chromatography, as well as mass spectrometry approaches (11). Among these analytical approaches, LC-MS/MS is extensively applied in lipidomics, particularly for identifying untargeted lipid profiles, due to its high resolution and preciseness (12). A previous LC-MS/MS study reported numerous differential mediumchain lipid species, including phosphatidylethanolamine (PE), phosphatidylcholine, diacylglycerol (DG), and prostaglandin (PG), between human milk and bovine or caprine milk (13). However, few studies have investigated the lipid profiles of preterm and term human milk using LC-MS/MS. Therefore, the identification and clarification of lipid expression profiles in preterm and term human milk are beneficial for understanding the protective impact of human milk on infants.

In this study, we aimed to assess differences in the lipidomic profiles of preterm, as well as term milk and to estimate the promising effects of these differential lipids on the development of neonates.

\section{METHODS}

\section{Sample Collection and Ethics Statement}

We collected colostrum samples from healthy lactating mothers who had given birth to either term (37-41 weeks), or preterm infants (32-36 weeks) at the Women's Hospital of Nanjing Medical University. Informed consent was submitted by the mothers of all the infants. The Human Research Ethics Committee of Women's Hospital of Nanjing Medical University ratified this study (Permission Number [2015] 88). Six of the mothers were assigned to the preterm group, and the other six mothers were assigned to the term group.

\section{Metabolite Extraction}

We transferred 100 microliters of each sample to a tube, and added $480 \mu \mathrm{L}$ of extract solution (MTBE: methanol $=5$ : 1). Subsequently, we sonicated the samples for $10 \mathrm{~min}$, then followed this with $1 \mathrm{~h}$ incubation at $-40^{\circ} \mathrm{C}$, and then $15 \mathrm{~min}$ centrifugation at $3,000 \mathrm{rpm}, 4^{\circ} \mathrm{C}$. A total of $350 \mu \mathrm{L}$ of supernatant was further transferred to a fresh tube, followed by drying at $37^{\circ} \mathrm{C}$ in a vacuum concentrator. We reconstituted the dried samples in $100 \mu \mathrm{L}$ of $50 \%$ methanol in dichloromethane. Then, we centrifuged the constitution at $12,000 \mathrm{rpm}$ for $15 \mathrm{~min}$ at $4^{\circ} \mathrm{C}$, and then transferred $75 \mu \mathrm{L}$ of the supernatant into a glass vial for LCMS/MS analysis. We prepared the quality control sample through blending equal aliquots of the supernatants of all the samples.

\section{LC-MS/MS Evaluation}

We accomplished UHPLC dissolution on a 1,290 Infinity series UHPLC system (Agilent Technologies), fitted with a Kinetex C18 column $(2.1 * 100 \mathrm{~mm}, 1.7 \mu \mathrm{m}$, Phenomen). The mobile phase A constituted water (40\%), acetonitrile (60\%), and ammonium formate $(10 \mathrm{mmol} / \mathrm{L})$ while the mobile phase $\mathrm{B}$ comprised of acetonitrile (10\%) and isopropanol (90\%), which included $50 \mathrm{~mL}$ of ammonium formate $(10 \mathrm{mmol} / \mathrm{L})$ for every $1,000 \mathrm{~mL}$ of the mixed solvent. We conducted the analysis by employing the following elution gradient: $0-12.0 \mathrm{~min}, 40-100 \%$ B; $12.0-13.5 \mathrm{~min}, 100 \% \mathrm{~B}$; $13.5-13.7 \mathrm{~min}, 100-40 \% \mathrm{~B}$; and $13.7-$ $18.0 \mathrm{~min}, 40 \% \mathrm{~B}$. The column temperature was $45^{\circ} \mathrm{C}$. We set the autosampler temperature and the injection volume at $4^{\circ} \mathrm{C}$, and 2 $\mu \mathrm{L}$ (pos) or $2 \mu \mathrm{L}$ (neg), respectively.

We utilized a triple TOF mass spectrometer because of its capacity to realize MS/MS spectra on data-dependent rationale during an LC/MS assay. In this mode, the acquisition software (Analyst TF 1.7, AB Sciex) continually assessed the entire scan survey MS information as it acquired and stimulated the collection of MS/MS spectra subject to the preselected maxims. During every cycle, the most intense 12 precursor ions with intensities higher than 100 were selected for MS/MS at a collision energy of $45 \mathrm{eV}$ (12 MS/MS events with accumulation time of $50 \mathrm{~ms}$ each). We set the ESI source parameters as: gas 1, 60 psi; gas 2, 60 psi; curtain gas, 30 psi; source temperature, $600^{\circ} \mathrm{C}$; declustering potential, $100 \mathrm{~V}$; and ion spray voltage floating, 5,000 or $-4,500 \mathrm{~V}$ in positive or negative modes, respectively.

\section{Statistical Analysis}

Using $\mathrm{R}$, an in-house algorithm called LipidAnalyzer for automatic data analysis was developed (14). Employing the "msconvert" of the ProteoWizard (version 3.0.6150), we converted the raw data files into the mzXML format. After that, we fed the mzXML files into LipidAnalyzer for data analysis (15). Firstly, we employed the CentWave algorithm in XCMS to apply peak detection to the MS1 data. With the MS/MS array, lipid determination was attained via a spectral match by utilizing a local MS/MS spectral library. We applied the orthogonal partial least squares discriminant analysis (OPLS-DA) to the unit variance-scaled spectral data for visualizing the differences between preterm and term human milk. The coefficient loading plots and variable importance in projection (VIP) of the OPLS-DA model were employed in identifying the spectral variables accounting for sample dissolution. The VIP scores ranked the components according to their importance for the observed separation. Statistical distinctness was established using the Student's $t$-test, with $P$ $<0.05$ signifying significance (16). We conducted pathway assessments in MetaboAnalyst (http://www.metaboanalyst.ca/). Furthermore, ingenuity pathway analysis (IPA, http://www. qiagen.com/ingenuity/) was also applied to decipher the molecular interaction networks that are dysregulated in preterm and term human milk.

\section{RESULTS}

\section{Clinical Characteristics}

The clinical features of the enrolled study participants are indicated in Table 1. The gestational ages of the term group and the preterm group were $39.16 \pm 0.65$ and $34.02 \pm 0.71$ weeks in the term group and the preterm group, respectively $(P<$ 0.05). The birth weight of infants was remarkably lower in the 
TABLE 1 | Maternal and infant characteristics.

\begin{tabular}{|c|c|c|c|}
\hline Characteristics & Term & Preterm & $P$-value \\
\hline \multicolumn{4}{|c|}{ Infants' Characteristics at Birth } \\
\hline $\begin{array}{l}\text { Gestational age } \\
\text { (weeks) }\end{array}$ & $39.16 \pm 0.65$ & $34.02 \pm 0.71$ & $<0.001$ \\
\hline Birth weight (g) & $3240.00 \pm 306.46$ & $2360.00 \pm 246.09$ & $<0.001$ \\
\hline Birth length (cm) & $49.83 \pm 0.41$ & $46 \pm 1.67$ & $<0.001$ \\
\hline \multicolumn{4}{|c|}{ Maternal Characteristics } \\
\hline $\begin{array}{l}\text { Maternal age } \\
\text { (years) }\end{array}$ & $28.83 \pm 6.08$ & $30.83 \pm 4.71$ & 0.538 \\
\hline $\begin{array}{l}\text { BMl at birth } \\
\left(\mathrm{kg} / \mathrm{m}^{2}\right)\end{array}$ & $26.47 \pm 4.22$ & $25.49 \pm 3.28$ & 0.663 \\
\hline GDM & 0 & 0 & \\
\hline $\mathrm{PlH}$ & 0 & 0 & \\
\hline Smoking history & 0 & 0 & \\
\hline Alcoholism history & 0 & 0 & \\
\hline $\begin{array}{l}\text { Cesarean section } \\
(\%)\end{array}$ & 33.33 & 50 & $>0.999$ \\
\hline $\begin{array}{l}\text { Milk collection time } \\
\text { (postpartum days) }\end{array}$ & $4.17 \pm 1.72$ & $4.00 \pm 1.67$ & 0.868 \\
\hline
\end{tabular}

Values were represented as mean $\pm S D(n=6)$. Student $t$-test and chi-square test was used for statistical analysis.

BMI, body mass index; GDM, gestational diabetes mellitus; PIH, pregnancyinduced hypertension. preterm group $(2360.00 \pm 246.09 \mathrm{~g})$ than in the term group (3240.00 $\pm 306.46 \mathrm{~g}, P<0.05)$. The average birth length was $49.83 \pm 0.41 \mathrm{~cm}$ in the term group and $46 \pm 1.67 \mathrm{~cm}$ in the preterm group $(P<0.001)$. However, there were no remarkable differences in maternal age, body mass index, delivery type, or milk collection time between the term and preterm groups. Human milk samples were collected at $4.17 \pm 1.72 \mathrm{~d}$ (term group) and $4.00 \pm 1.67 \mathrm{~d}$ (preterm group, $P>0.05$ ). The subjects were healthy mothers without maternal complications or bad habits. Among them, three mothers gave birth prematurely due to premature rupture of the membrane, while one mother had central placenta previa. The cause of premature delivery of the other two mothers is unclear.

\section{Multivariate Statistical Analysis of Lipids}

Multivariate assessment, OPLS-DA, was employed to analyze the differences in preterm and term human milk lipids. OPLSDA constitutes a supervised trend identification approach that allows for sample classification and removes unassociated noise data from the dataset. OPLS-DA was determined to additionally verify the dissolution of the metabolic profiles between the term and preterm human milk arms. The parameters $R^{2} Y$, as well as $\mathrm{Q}^{2}$, were $>0.5$ in the positive and negative ionization modes, implying that the OPLS-DA model was well-determined. In the OPLS-DA score plots, the samples from the term and preterm human milk arms were completely separated (Figures 1A,C),
A

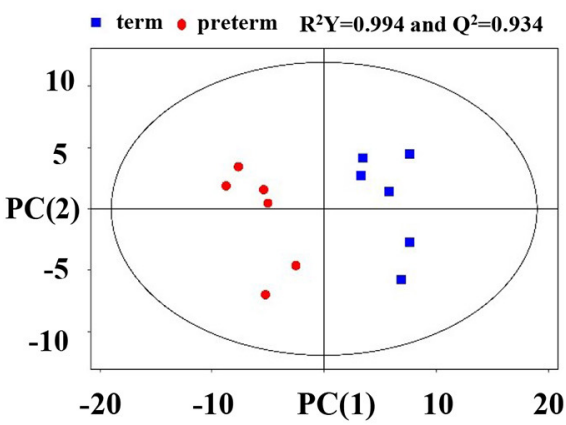

C

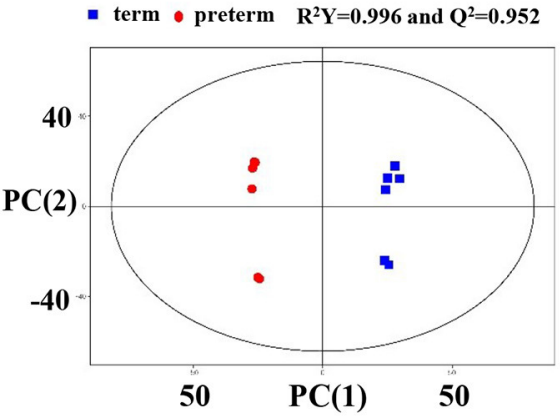

B

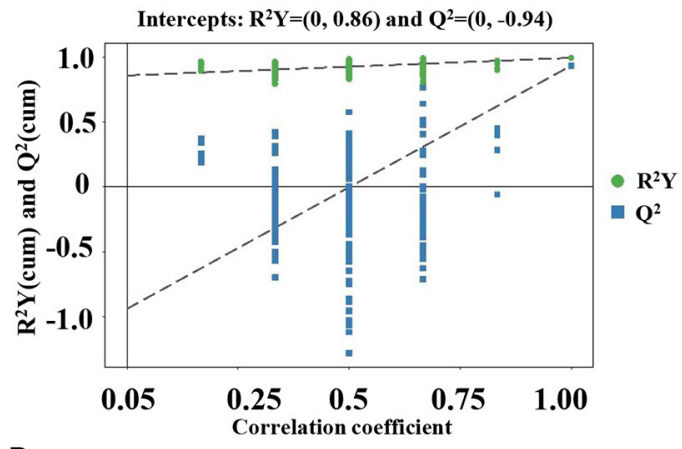

D

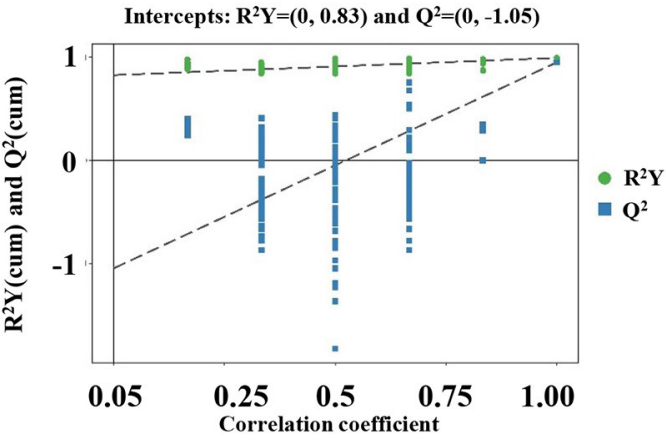

FIGURE 1 | Score scatter plot and permutation test of the OPLS-DA model. (A) Score plots were generated from the LC-MS/MS data of preterm and term human milk lipidomic profiles in the positive ionization mode. The $x$ axis and $y$ axis represent the first and second principle components, respectively. The red and blue colors indicate respective groups. (B) Permutation tests were obtained from LC-MS/MS data in the positive ionization mode. The $x$ axis and $y$ axis of the permutation test represent the correlation coefficient and value of $R^{2} Y$ and $Q^{2}$, respectively. (C) Score plots were obtained in the negative ionization mode. (D) Permutation tests were obtained in the negative ionization mode. OPLS-DA, orthogonal partial least-squares discriminant analysis. 
further positing that there were distinct differences in the metabolic features of human milk. A permutation examination of the OPLS-DA model was proceeded to further verify the model. The $R^{2} Y$ and $Q^{2}$ intercept values were $(0,0.86)$ and $(0,-0.94)$, respectively, in the positive ionization mode (Figure 1B), and $(0,0.83)$ and $(0,-1.05)$, respectively, in the negative ionization mode (Figure 1D). The results showed that $\mathrm{R}^{2} \mathrm{Y}$ and $\mathrm{Q}^{2}$ were the greatest in the current sample clustering, implying that our OPLS-DA model was robust, without overfitting. The low $\mathrm{Q}^{2}$ intercept values demonstrated the robustness of the model, and thus revealed authenticity, as well as a low risk of overfitting.

\section{Different Lipids Observed in Preterm and Term Human Milk}

An LC-MS/MS strategy was used to determine the lipid profiles of preterm, as well as term human milk. Based on the results of the LC-MS/MS analysis, 4,686 lipids were found (Figure 2A). After eliminating the lipids identified in both the positive, as well as the negative ion mode repeatedly, 235 lipids (VIP $>1, p<$ 0.05 ) were remarkably different when comparing the preterm and term human milk groups, including 131 upregulated lipids and 104 downregulated lipids in the preterm group (Figure 2B). The top 30 markedly up modulated and down modulated lipids in the preterm arm can be found in Table 2. All of the markedly different lipids are shown in Supplementary Table 1.

\section{Preterm Human Milk Contains Higher Level of PE and PC Than Term Human Milk}

The differences in the comparative proportion of identified lipid species were used to reveal the composition and distribution of lipids. The relative differences among the lipid clusters exhibited remarkable changes in the levels of lipid species in most of the inspected lipid groups. In total, 16 lipid subclasses, including triacylglycerol (TG), sphingosine, sphingomyelins
(SM), phosphatidylserine (PS), phosphatidylinositol (PI), PG, PE, phosphatidylcholine (PC), monoacylglycerol (MG), DG, cardiolipin (CL), cholesteryl ester (CE), hexosylceramide (HexCer), dihexosylceramide (Hex2Cer), ceramide phosphate (CerP), and ceramide (Cer) were significantly different between the preterm and term arms (Figures 3A,B). Interestingly, $\mathrm{PE}, \mathrm{PC}, \mathrm{DG}$, and Cer changed robustly in the two groups (Figure 3B). The top three significantly upregulated PE in the preterm arm includes PE (22:1/0:0), PE (26:2/14:1), and PE (24:1/18:1) (Figure 4A). The top three significantly upregulated PC in the preterm arm includes PC (14:0/22:5), PC (10:0/26:2), and PC (11:0/22:5) (Figure 4B). The top three significantly downregulated DG in the preterm arm includes DG (22:6/22:2), DG (16:0/11:0), and DG (18:1/12:0) (Figure 4C). The top three significantly downregulated Cer in the preterm arm includes Cer (d17:1/22:0), Cer (t15:1/24:0), and Cer (d18:1/18:1) (Figure 4D).

\section{The Metabolomic Pathways of the Differential Lipids Between the Term and Preterm Groups}

Pathway analysis by MetaboAnalyst was conducted to reveal the potential functions of the significantly different lipids. These lipids were predicted to participate in eight metabolic pathways, including glycerophospholipid biometabolism, glycosylphosphatidylinositol-anchor biosynthesis, glycerolipid biometabolism, ether lipid biometabolism, sphingolipid biometabolism, linoleic acid biometabolism, alpha-linolenic acid biometabolism, and arachidonic acid biometabolism (Figure 5). Additionally, the dysregulated lipids were loaded into the IPA software for biological cascade assessments. The findings of the IPA evaluation indicated that these lipids were involved in the Reelin signaling in neurons and in LXR/RXR activation (Figure 6).
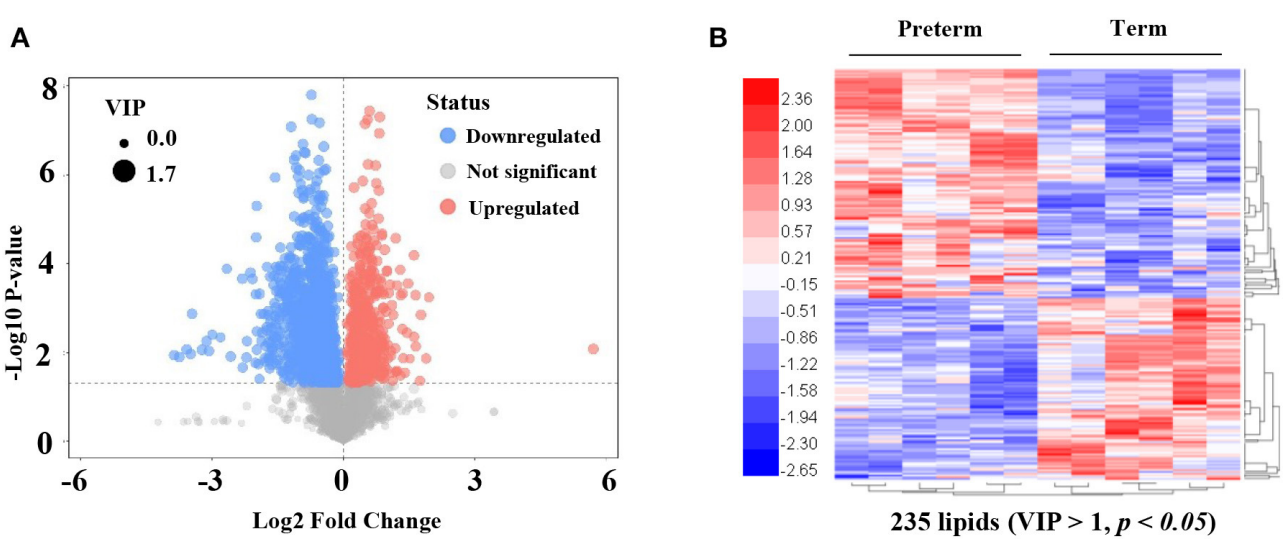

FIGURE 2 | Significantly different lipids in preterm and term human milk. (A) The volcano plot is used for assessing significantly different lipids. Blue dots indicate downregulated lipids in the preterm group; red dots indicate significantly upregulated lipids in the preterm group; gray dots indicate the lipids that were not significantly changed in the preterm group. The size of dots indicates VIP value. (B) The heatmap of hierarchical clustering analysis is used for assessing significantly upregulated and downregulated lipids. Increased and decreased lipid levels are depicted by red and blue colors, respectively. The dendrogram was constructed based on the lipid intensity. VIP, variable importance in projection. 
TABLE 2 | Top 30 significantly upregulated and downregulated lipids in preterm group.

\begin{tabular}{|c|c|c|c|c|}
\hline Name & Preterm & Term & VIP & $P$-value \\
\hline \multicolumn{5}{|l|}{ Upregulated } \\
\hline HexCer(d18:1/16: & 8.67274E-05 & 5.72774E-05 & 1.736105792 & 3.6502E-08 \\
\hline CerP(d18:1/24 & & & 1.711457827 & \\
\hline PE(24:1/10:0) & 0.000404915 & 0.0002426 & 1.715919004 & $6.17433 \mathrm{E}-0$ \\
\hline PE(P-18:0/22 & 9.90662E-05 & 7.00258E-05 & 1.680976896 & $5.75733 \mathrm{E}$ \\
\hline E(22:2/18: & 8 & $0.00017 \varepsilon$ & 1.679793 & \\
\hline$C(16: C$ & & & & \\
\hline $4: 4)$ & 0.00052 & 0.00037 & 1.64813 & \\
\hline PE(26:0/6:0) & 2.81898E-05 & $2.13804 \mathrm{E}-05$ & 1.63344 & $2.37242 \mathrm{E}$ \\
\hline $\mathrm{PC}(11: 0$, & 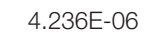 & 1.84 & 1.66179 & 2.6 \\
\hline$E(22: 5 / 16: 1)$ & 0.000129993 & & 1.656433 & \\
\hline$P C(14: 1 / 24$ & 7.79234E & $4.79878 \mathrm{E}$ & 1.635165 & $3.04858 \mathrm{E}-05$ \\
\hline TG(18:1/18:1/ & 0.029991392 & 0.026391 & 1.642302 & $4.08878 \mathrm{E}-0$ \\
\hline$G(16: 1 / 16: 1 / 2$ & 0.003456 & 0.00 & 1.643 & \\
\hline I(14:1/22:2) & 70714E-05 & & & \\
\hline TG(18:2/18:2 & 0.021745766 & 0.016665 & 1.643068 & 5.82 \\
\hline$C(17: 0$ & 2.48972E-05 & 1.89 & 1.62 & 5 \\
\hline c(22 & 022 & 9.7 & & 8.3 \\
\hline$C(18: 2 / 22: 6)$ & 4571E & 9.05764 & 1 & \\
\hline & & & & \\
\hline A(16:1 & 0.016 & 0.01 & 1.58392 & 0.00 \\
\hline PE(8:0/26:2) & 80808E-06 & 7.50189E-06 & 1.587356621 & $0.00013037 \varepsilon$ \\
\hline TG(18:2/18:2/18:3) & & & & \\
\hline TG(16:1/18:1/18:2) & 0.037039637 & & 1.58536 & 0.00 \\
\hline$E(24:$ & 8.19788E-05 & 5.89 & 1.52823 & 0.00 \\
\hline 22:6) & $36971 \mathrm{E}$ & 5.21967E-06 & 1.471141 & 0.000218238 \\
\hline ) & 6 & & & 1819 \\
\hline$P E(P-16:$ & & & & \\
\hline $\mathrm{PC}(17: 0$ & 3.33652E-05 & $2.0138 \mathrm{E}-05$ & 1.662093778 & 0.000 \\
\hline PE(22:4/18:3) & 0.000299337 & 0.000227105 & 1.5865266 & 0.001929724 \\
\hline HexCer(d18:1/16:0) & & & & \\
\hline \\
\hline MG(18: & 6.68287E-05 & 0.00 & 1.6983895 & 3.23597E-07 \\
\hline MG(22 & 499E-05 & $.13336 \mathrm{E}$ & 1.717901 & 3.69537E-07 \\
\hline $\mathrm{DG}(18: 1$ & 003802847 & 0.006430644 & 1.67435 & 9.98 \\
\hline & & & & \\
\hline DG(18:1/11:0) & 2.86785E-05 & 4.03112E-05 & 1.667830547 & 1.83293E-0 \\
\hline PC(P-20:0/18: & 63191E-05 & 3.34275E-05 & 1.583959212 & $2.60475 \mathrm{E}-05$ \\
\hline 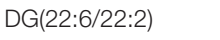 & & & & \\
\hline & & & & \\
\hline SM(d15:0/20:0) & 7.81324E-06 & 9.92859E-06 & 1.573560159 & $7.48258 \mathrm{E}-05$ \\
\hline TG(14:0/16:0/16:0) & 0.0002281 & 0.000314764 & 1.528791154 & 0.000102201 \\
\hline$P(1-2<0$ & 5.95593E-0 & & 1.562153134 & 0.000163043 \\
\hline & & & & 0.000168646 \\
\hline PS(20:0/15:1) & 7.17214E-06 & $1.68524 \mathrm{E}-05$ & 1.610366809 & 0.000250386 \\
\hline 16:0/18:3) & 2.74301E-05 & 3.3855E-05 & 1.531444256 & 0.000352698 \\
\hline PE(22: & $3281 \mathrm{E}-$ & 5.11025E-06 & 1.674753549 & 0.00043549 \\
\hline$P E(P-16: 0 / 22: 5)$ & 1.20013E-C & $2.08918 \mathrm{E}-$ & 1.545456353 & 0.00044156 \\
\hline & 3.8249E-07 & 5.66847E-07 & 1.495903496 & 0.000446668 \\
\hline PC(O-18:1/0:0) & 5.35639E-06 & $9.10166 \mathrm{E}-06$ & 1.660464722 & 0.00044843 \\
\hline
\end{tabular}

(Continued)
TABLE 2 | Continued

\begin{tabular}{lcccc}
\hline Name & Preterm & Term & VIP & P-value \\
\hline TG(17:1/17:1/19:0) & 0.000253649 & 0.00031972 & 1.501064831 & 0.000473077 \\
DG(18:1/12:0) & 0.000623169 & 0.001082651 & 1.551640966 & 0.000486342 \\
DG(18:0/18:0) & $4.21601 \mathrm{E}-05$ & $5.76514 \mathrm{E}-05$ & 1.650211743 & 0.000550865 \\
$\mathrm{DG}(20: 1 / 20: 1)$ & $5.96911 \mathrm{E}-05$ & $6.98941 \mathrm{E}-05$ & 1.417417102 & 0.00089955 \\
$\mathrm{TG}(20: 1 / 20: 1 / 20: 3)$ & $5.3131 \mathrm{E}-06$ & $8.60194 \mathrm{E}-06$ & 1.458157757 & 0.000925138 \\
$\mathrm{TG}(12: 0 / 16: 0 / 18: 0)$ & 0.00174576 & 0.002266135 & 1.498999123 & 0.000963483 \\
$\mathrm{PS}(22: 2 / 14: 0)$ & 0.00097586 & 0.001104228 & 1.441677973 & 0.00109107 \\
$\mathrm{DG}(16: 0 / 16: 0)$ & 0.001142656 & 0.001410798 & 1.599660866 & 0.001113963 \\
$\mathrm{TG}(21: 0 / 22: 1 / 22: 2)$ & $6.95148 \mathrm{E}-06$ & $1.31767 \mathrm{E}-05$ & 1.423852611 & 0.001177453 \\
$\mathrm{MG}(14: 0)$ & $3.88534 \mathrm{E}-06$ & $7.44905 \mathrm{E}-06$ & 1.625605223 & 0.001185778 \\
$\mathrm{TG}(20: 3 / 22: 4 / 22: 4)$ & $1.00909 \mathrm{E}-05$ & $2.05045 \mathrm{E}-05$ & 1.404088604 & 0.001327215 \\
$\mathrm{PC}(2: 0 / 24: 0)$ & $1.41719 \mathrm{E}-06$ & $2.69187 \mathrm{E}-06$ & 1.50878231 & 0.001420451
\end{tabular}

Preterm, mean value of preterm group; Term, mean value of term group; VIP, variable importance in projection.

\section{DISCUSSION}

Human milk provides essential nutrients and bioactive factors to support the organ development and immune system of newborns $(2,17)$. Colostrum, the fluid produced by the mammary glands over the first few postnatal days, is rich in biologically protective components; these components are present in elevated levels in the colostrum of mothers who have given birth to preterm infants than in that of mothers who have given birth to term infants (18). Additionally, mature milk becomes stable to avoid further fluctuations $(19,20)$. Human milk lipids are crucial sources of biological components like choline and long-chain polyunsaturated fatty acids important for neural and visual development (8). Herein, we examined the composition of lipids in the colostrum of mothers who gave birth at different gestational ages. LC-MS/MS, a powerful analytical method, was applied to identify and clarify a large number of lipids in preterm, as well as term human milk to understand the protective effects of human milk on infants.

According to the LC-MS/MS results, the constitution of colostrum lipids was found to vary at different gestational ages. Overall, 16 sub-clusters of 235 lipids were significantly different in the preterm and term human milk arms. In contrast to other traditional approaches, LC-MS/MS enabled concurrent determination and quantitation of hundreds of significantly different lipids in preterm and term human milk $(8,21)$. Notably, PE and PC were increased robustly in preterm human milk, while DG and Cer were decreased robustly in preterm human milk. PE, as an important constituent of mammalian cell membranes, serves a critical role in biological processes, including apoptosis, as well as cell signaling (22). Moreover, PE can also enhance memory and facilitate brain function development (23). Therefore, the high levels of remarkably different PE, including PE (22:1/0:0), PE (26:2/14:1), and PE (24:1/18:1), in preterm human milk may be strongly correlated with nervous system development in infants. PC is considered to be highly important in the development of infants, since the 


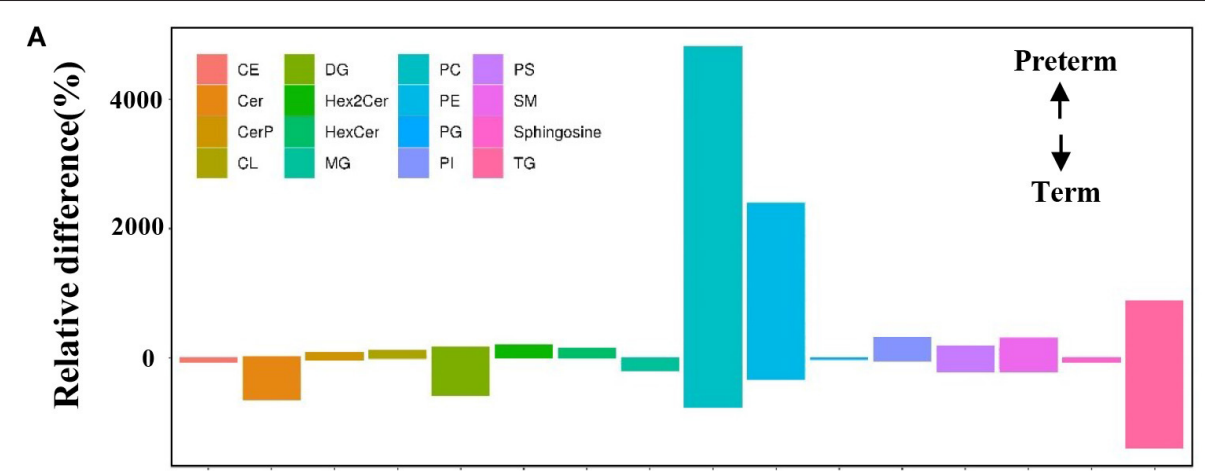

B

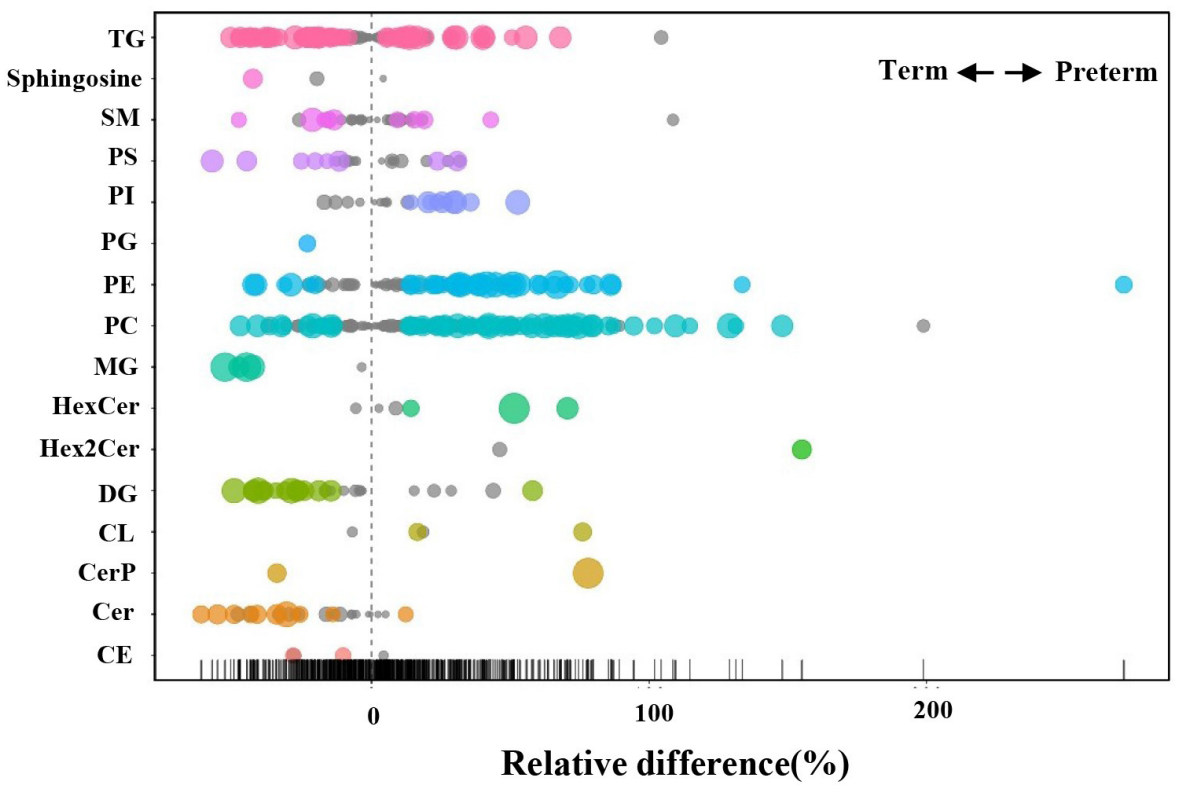

FIGURE 3 | Differences in the relative percentage of significantly different lipid species between preterm and term human milk. (A) The bar plot shows the relative difference in the detected significantly different lipids. Each column represents a subclass of lipids. The y axis represents the relative difference of various lipids. A positive relative difference indicates that the content of this kind of substance was higher in the preterm group. A negative relative difference indicates that the content of this kind of substance was higher in the term group. The values corresponding to the top and bottom margins of the column represent the relative difference. (B) A bubble plot shows the relative differences in the detected lipids. Each point represents a lipid. The size of the point represents the $P$-value. Larger points represent smaller $P$-values. Colored points indicate that the $P<0.05$.

choline present in the neonates partly comes from PC (24). An increased choline requirement of preterm infants was reported in a previous study (25). Choline deficiency may lead to impaired neurocognitive and pulmonary development in preterm infants (25). The choline-triggered spatial memory enhancement was reported to correlate with variations in the birth, apoptosis, and migration of cells in the hippocampus during the development of the brain (26). Typically, choline translocation is introduced from the liver to other organs. In choline insufficiency, nonetheless, it is reverted toward the liver to maintain the hepatic providences, at the cost of the lungs. This may especially impact the developing lung (25). Except for being the choline source, PC facilitates the liver to regenerate from toxicity via the donation of methyl groups for hepatic recovery (27). Additionally, similar amounts of PC were added to infant formulas generated from bovine milk (28). These results suggest that PC, such as PC (14:0/22:5), PC (10:0/26:2), and PC (11:0/22:5), may serve pivotal roles in preterm infant development. DG, one of the primary lipid subgroups in living systems and a second messenger in multiple cell activities, has been reported to hasten the $\beta$-oxidation of fatty acids, as well as influence the expression of lipid metabolismlinked genes, thereby diminishing TG levels in the serum, as well as in the liver $(29,30)$. The high level of DG, such as DG (22:6/22:2), DG (16:0/11:0), and DG (18:1/12:0), in term human milk may be linked to enhanced long-term health outcomes; for instance, reduced obesity, diabetes, and cardiovascular disease. 
A

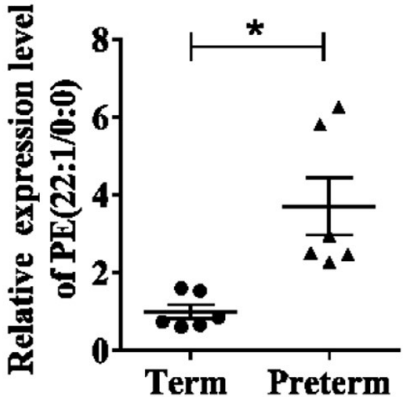

B

c
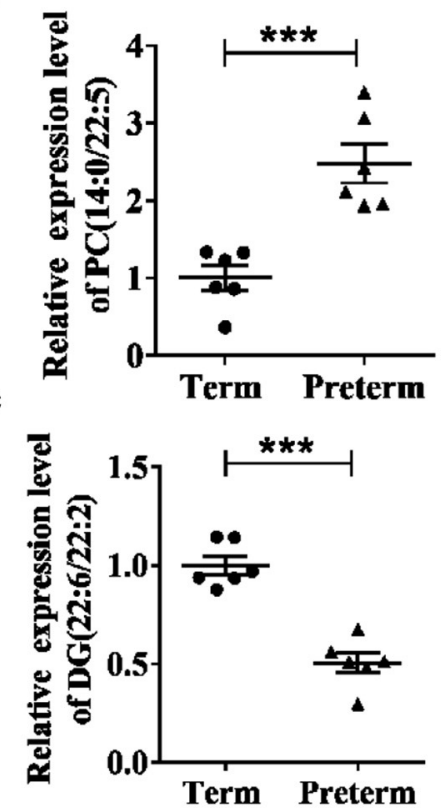

D

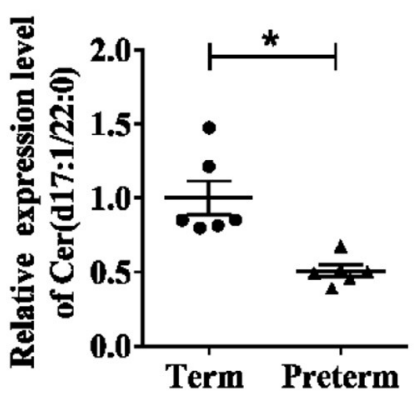

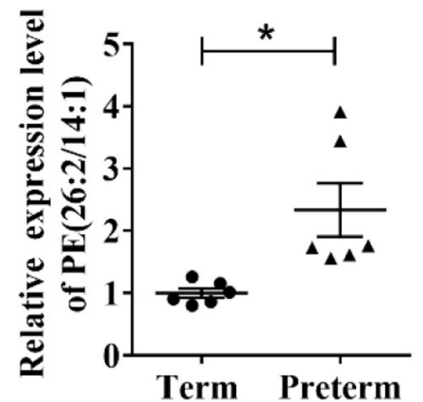
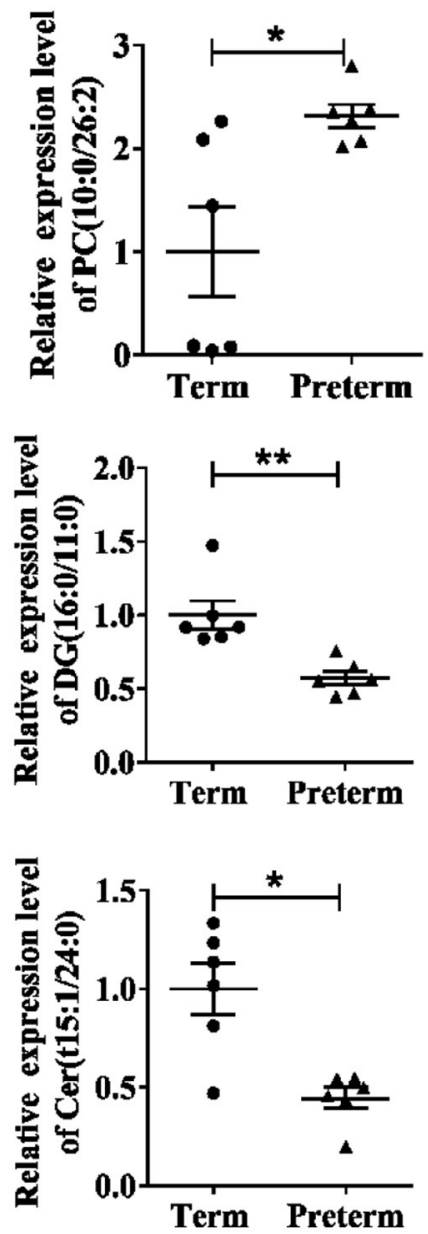
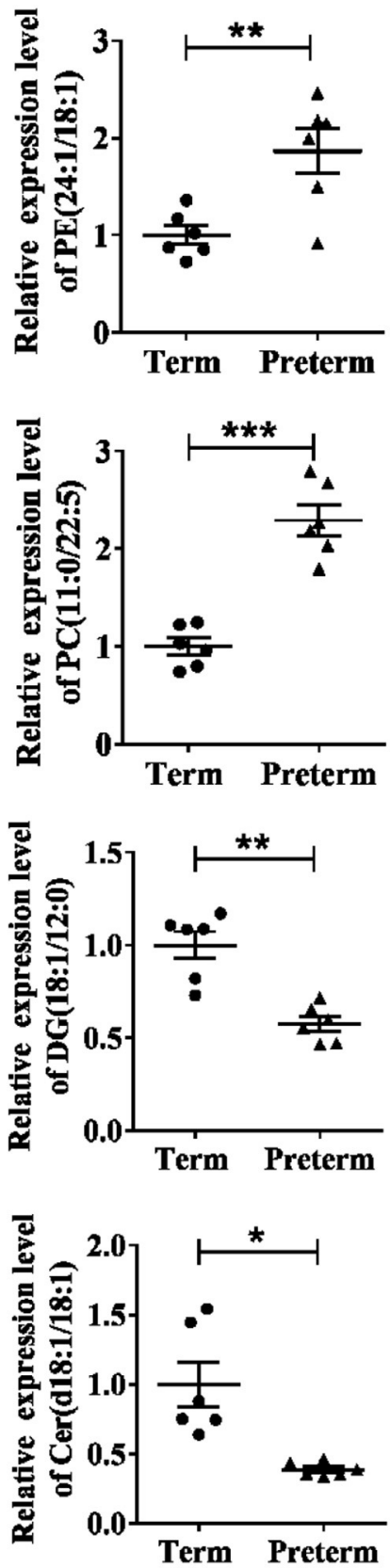

FIGURE 4 | Twelve significantly different lipids in the term and preterm groups. (A) Top three significantly upregulated PEs in preterm group. (B) Top three significantly upregulated PCs in preterm group. (C) Top three significantly downregulated DGs in preterm group. (D) Top three significantly downregulated Cers in preterm group. PE, phosphatidylethanolamine; PC, phosphatidylcholine; DG, diacylglycerol; Cer, ceramide. ${ }^{*} P<0.05$; ${ }^{* \star} P<0.01$; ${ }^{\star \star \star} P<0.001$.

Cer has many signaling roles linked to the modulation of cell growth and the initiation of apoptosis (31). In the mature, as well as the neonatal gut of term infants, mature villus cells go through apoptosis during mucosal regeneration and Cer may influence this process (32). Our data suggest that these changed subclasses of lipids may be beneficial for understanding the protective effects of preterm and term breast milk on infants.
To gain a better insight into the protective function of human milk lipids, we conducted a pathway examination to reveal the potential mechanism. Notably, the results of the pathway analysis demonstrated that the different lipids were associated with glycerophospholipid biometabolism, glycosylphosphatidylinositol-anchor biosynthesis, glycerolipid biometabolism, ether lipid biometabolism, and sphingolipid 


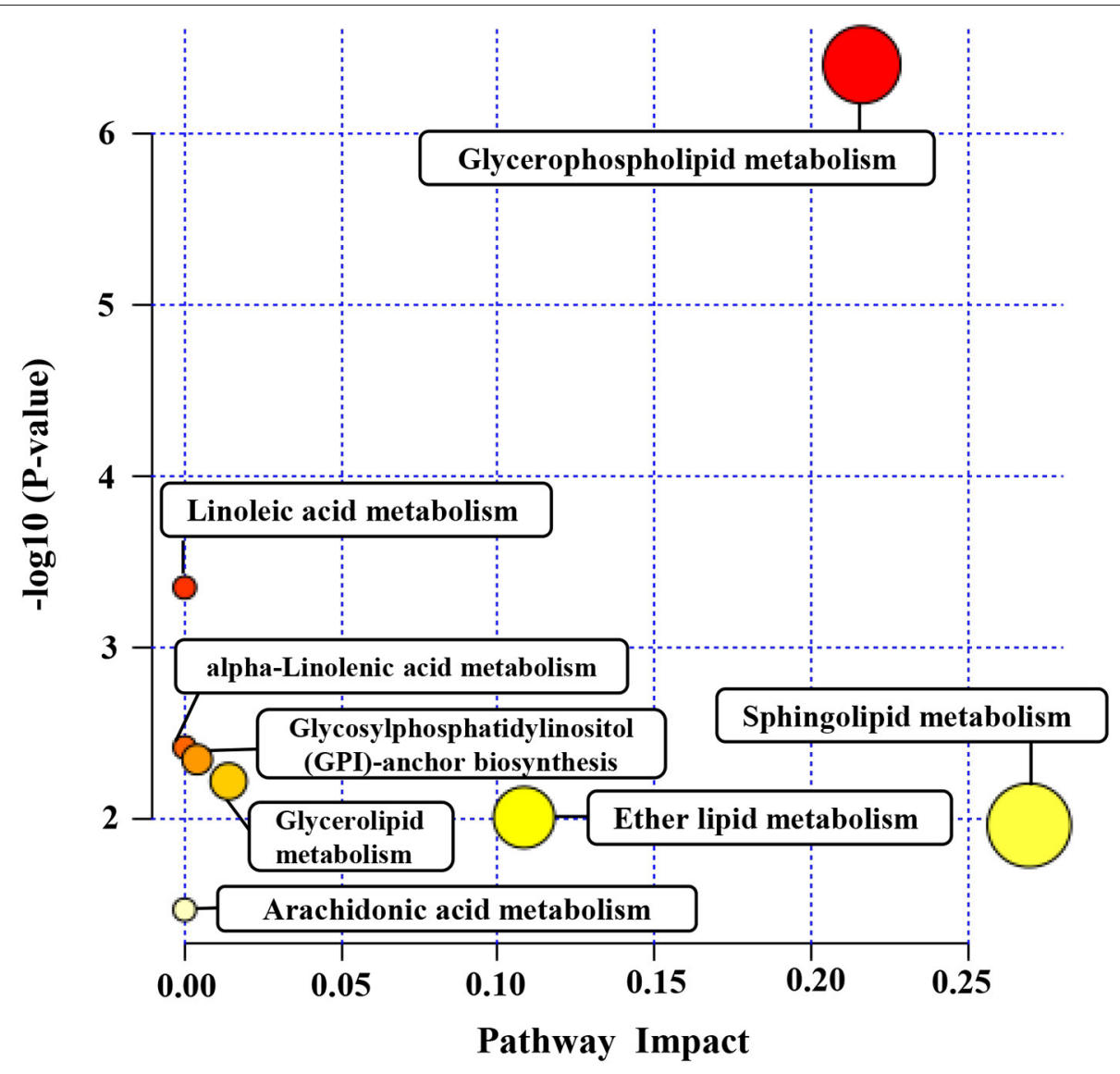

FIGURE 5 | The metabolomic pathway of significantly different lipids analyzed by MetaboAnalyst. Pathway analysis of the significantly different lipids was carried out by MetaboAnalyst. The y axis represents the transformation of the original $P$-value calculated from the enrichment analysis. The $x$ axis represents the value calculated from the pathway topology analysis.

biometabolism. Glycerophospholipid metabolism and sphingolipid metabolism were identified as the most pivotal metabolic cascades with an impact value $>0.2$. Glycerophospholipid metabolism was reported to serve a crucial role in the regulation of monocytes and macrophages (33). The critical roles of monocytes and macrophages are the elimination of microorganisms, toxic particles, and damaged cells from inflammation sites (34). Moreover, glycerophospholipid biometabolism, a part of lipid biometabolism, participates in the PE and PC biosynthesis, which are both important for infants as described above. We speculate that glycerophospholipid metabolism may participate in the lipid dysregulation of preterm as well as term human milk. Sphingolipids are constituents of the plasma membrane and are considered regulators of cell-cell interactions, as well as cell recognition (35). Sphingolipid metabolism pathways were reported to be vital targets for therapeutics development $(36,37)$. A variety of studies have recently shown that affecting the enzymes involved in these sphingolipid biosynthetic pathways using inhibitors or gene deletion can attenuate the virulence of fungal pathogens; for instance, Candida albicans, Cryptococcus neoformans, and
Aspergillus spp (38-40). Some key sphingolipid metabolites, including fatty acid 2-hydroxylase, ACER3, and ceramide synthase 2 have been proven to be critical for normal brain development and function (41-43). Notably, Reelin signaling was reported to control diverse phases of neuronal migration in the developing brain, including terminal translocation, the transition from the multipolar to bipolar neurons, and the termination of migration underneath the marginal zone (44-46). Defects in neuronal migration are thought to be involved in multiple neurological disorders, such as mental retardation (47). We speculate that Reelin signaling may participate in the improvement of neurodevelopmental outcomes for infants mediated by human milk. The integrated activation of LXR and RXR was shown to promote innate immunity to bacterial pathogens by their potential to protect macrophages from cell death caused by infection with Escherichia coli (48). LXR/RXR activation may provide a possible explanation for the antiinfection potential of human milk. However, these pathways need to be further validated.

In summary, the lipids in preterm and term colostrum were identified by LC-MS/MS. Significantly different lipidomic 


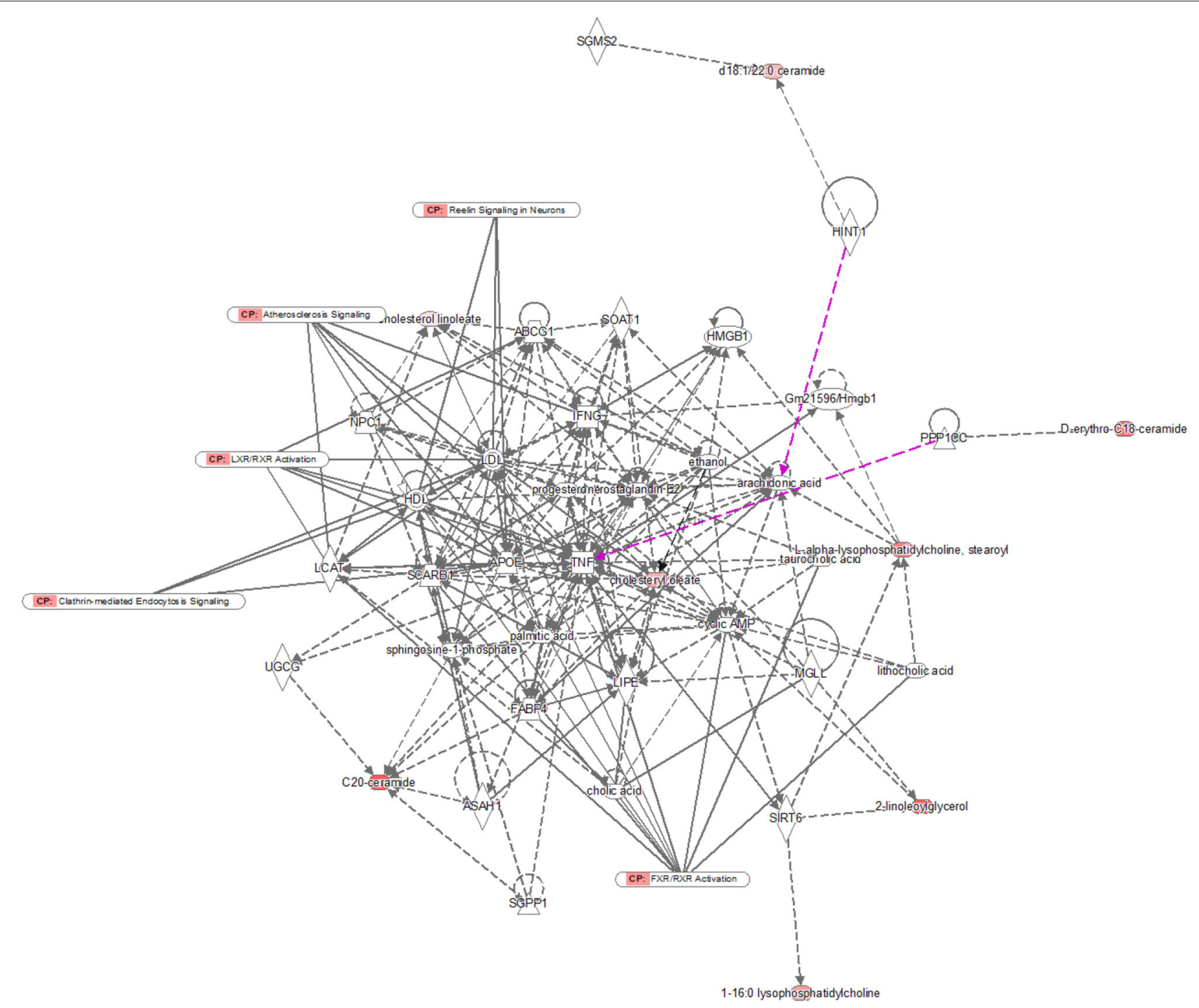

FIGURE 6 | IPA prediction networks of significantly different lipids. The significantly different lipids were imported into the IPA software for pathway analysis. Direct connections are shown via solid lines, while indirect interactions are shown via dashed lines. IPA, ingenuity pathway analysis.

profiles and a variety of different lipids between preterm and term human milk were observed. Notably, PE and PC were increased robustly in preterm human milk, while DG and Cer were decreased robustly in preterm human milk. The significantly different lipids are involved in various pathways, including glycerophospholipid metabolism, sphingolipid metabolism, Reelin signaling in neurons, and LXR/RXR activation. These findings suggest that the presence of different lipids may explain the different effects of preterm and term human milk on infants. Our findings may provide novel insights into the mechanism of human milk lipids in neonatal development. It may offer pivotal information for the utilization of these critical constituents as nutritional, as well as functional factors in infant formula. Further studies should aim at increasing the sample size to elucidate the detailed biomechanism of these dysregulated lipids.
Moreover, further studies should be conducted to validate these lipids and metabolic pathways.

\section{DATA AVAILABILITY STATEMENT}

The data that support the findings of this study are available from the corresponding author upon reasonable request.

\section{ETHICS STATEMENT}

The studies involving human participants were reviewed and approved by Human Research Ethics Committee of Women's Hospital of Nanjing Medical University [Permission Number (2015) 88]. The patients/participants provided their written informed consent to participate in this study. 


\section{AUTHOR CONTRIBUTIONS}

LX, WC, XW, and ZY: collected and analyzed the data. SH: designed the study and wrote the manuscript. All authors approved the final submission.

\section{FUNDING}

This work was funded by grants from the National Natural Science Foundation of China (Grant No. 81971427), the National Natural Science Foundation for the Youth

\section{REFERENCES}

1. Gartner LM, Morton J, Lawrence RA, Naylor AJ, O’Hare D, Schanler RJ, et al. Breastfeeding and the use of human milk. Off J Am Acad Pediatrics. (2005) 129:496-506. doi: 10.1542/peds.2004-2491

2. Boquien CY. Human milk: an ideal food for nutrition of preterm newborn. Front Pediatr. (2018) 6:295. doi: 10.3389/fped.2018.00295

3. Lewis ED, Richard C, Larsen BM, Field CJ. The importance of human milk for immunity in preterm infants. Clin Perinatol. (2017) 44:2347. doi: 10.1016/j.clp.2016.11.008

4. Corpeleijn WE, Kouwenhoven SM, Paap MC, van Vliet I, Scheerder I, Muizer Y, et al. Intake of own mother's milk during the 1st days of life is associated with decreased morbidity and mortality in very low birth weight infants during the first 60 days of life. Neonatology. (2012) 102:27681. doi: $10.1159 / 000341335$

5. Lechner BE, Vohr BR. Neurodevelopmental outcomes of preterm infants fed human milk: a systematic review. Clin Perinatol. (2017) 44:6983. doi: 10.1016/j.clp.2016.11.004

6. Lucas A. Long-term programming effects of early nutritionimplications for the preterm infant. J Perinatol. (2005) 25(Suppl.2):S26. doi: $10.1038 /$ sj.jp.7211308

7. Gidrewicz DA, Fenton TR. A systematic review and meta-analysis of the nutrient content of preterm and term breast milk. BMC Pediatr. (2014) 14:216. doi: 10.1186/1471-2431-14-216

8. Ingvordsen Lindahl IE, Artegoitia VM, Downey E, O’Mahony JA, O'Shea CA, Ryan CA, et al. Quantification of human milk phospholipids: the effect of gestational and lactational age on phospholipid composition. Nutrients. (2019) 11:20222. doi: 10.3390/nu11020222

9. Koletzko B. Human milk lipids. Ann Nutr Metab. (2016) 69(Suppl.2):2840. doi: 10.1159/000452819

10. Ibáñez C, Mouhid L, Reglero G, Ramírez de Molina A. Lipidomics insights in health and nutritional intervention studies. J Agric Food Chem. (2017) 65:7827-42. doi: 10.1021/acs.jafc.7b02643

11. George AD, Gay MCL, Trengove RD, Geddes DT. Human milk lipidomics: current techniques and methodologies. Nutrients. (2018) 10:91169. doi: 10.3390/nu10091169

12. Leung KS, Fong BM. LC-MS/MS in the routine clinical laboratory: has its time come? Anal Bioanal Chem. (2014) 406:2289-301. doi: 10.1007/s00216-013-7542-5

13. Wang L, Li X, Liu L, da Zhang H, Zhang Y, Hao Chang Y, et al. Comparative lipidomics analysis of human, bovine and caprine milk by UHPLCQ-TOF-MS. Food Chem. (2020) 310:125865. doi: 10.1016/j.foodchem.201 9.125865

14. Zhou Z, Shen X, Chen X, Tu J, Xiong X, Zhu ZJ. LipidIMMS Analyzer: integrating multi-dimensional information to support lipid identification in ion mobility-mass spectrometry based lipidomics. Bioinformatics. (2019) 35:698-700. doi: 10.1093/bioinformatics/bty661

15. Tu J, Yin $\mathrm{Y}, \mathrm{Xu} \mathrm{M}$, Wang $\mathrm{R}$, Zhu ZJ. Absolute quantitative lipidomics reveals lipidome-wide alterations in aging brain. Metabolomics. (2017) 14:5. doi: 10.1007/s11306-017-1304-x

16. Yan B, Chu H, Yang D, Sze KH, Lai PM, Yuan S, et al. Characterization of the lipidomic profile of human coronavirus-infected cells: implications for
Scholars of China (Grant No. 81901512), and the Science and Technology Development Fund of Nanjing Medical University (No. NMUB2018127).

\section{SUPPLEMENTARY MATERIAL}

The Supplementary Material for this article can be found online at: https://www.frontiersin.org/articles/10.3389/fped. 2020.00590/full\#supplementary-material

Supplementary Table 1 | List of the significantly different lipids.

lipid metabolism remodeling upon coronavirus replication. Viruses. (2019) 11:73. doi: $10.3390 / \mathrm{v} 11010073$

17. Gura T. Nature's first functional food. Science. (2014) 345:7479. doi: 10.1126/science.345.6198.747

18. Araújo ED, Gonçalves AK, Cornetta Mda C, Cunha H, Cardoso ML, Morais SS, et al. Evaluation of the secretory immunoglobulin A levels in the colostrum and milk of mothers of term and pre-term newborns. Braz J Infect Dis. (2005) 9:357-62. doi: 10.1590/S1413-86702005000500002

19. Castellote C, Casillas R, Ramírez-Santana C, Pérez-Cano FJ, Castell M, Moretones MG, et al. Premature delivery influences the immunological composition of colostrum and transitional and mature human milk. J Nutr. (2011) 141:1181-7. doi: 10.3945/jn.110.133652

20. Ballard O, Morrow AL. Human milk composition: nutrients and bioactive factors. Pediatr Clin North Am. (2013) 60:4974. doi: $10.1016 /$ j.pcl.2012.10.002

21. Bitman J, Wood DL, Mehta NR, Hamosh P, Hamosh M. Comparison of the phospholipid composition of breast milk from mothers of term and preterm infants during lactation. Am J Clin Nutr. (1984) 40:110319. doi: $10.1093 / \mathrm{ajcn} / 40.5 .1103$

22. Vance JE. Phosphatidylserine and phosphatidylethanolamine in mammalian cells: two metabolically related aminophospholipids. J Lipid Res. (2008) 49:1377-87. doi: 10.1194/jlr.R700020-JLR200

23. Modica-Napolitano JS, Renshaw PF. Ethanolamine and phosphoethanolamine inhibit mitochondrial function in vitro: implications for mitochondrial dysfunction hypothesis in depression and bipolar disorder. Biol Psychiatry. (2004) 55:273-7. doi: 10.1016/S0006-3223(03)00784-4

24. Zou XQ, Guo Z, Huang JH, Jin QZ, Cheong LZ, Wang XG, et al. Human milk fat globules from different stages of lactation: a lipid composition analysis and microstructure characterization. J Agri Food Chem. (2012) 60:715867. doi: 10.1021/jf3013597

25. Bernhard W, Poets CF, Franz AR. Choline and choline-related nutrients in regular and preterm infant growth. Eur J Nutr. (2019) 58:93145. doi: 10.1007/s00394-018-1834-7

26. Holmes-McNary MQ, Loy R, Mar MH, Albright CD, Zeisel SH. Apoptosis is induced by choline deficiency in fetal brain and in PC12 cells. Brain Res Dev Brain Res. (1997) 101:9-16. doi: 10.1016/S0165-3806(97)00044-8

27. Cilla A, Diego Quintaes K, Barberá R, Alegría A. Phospholipids in human milk and infant formulas: benefits and needs for correct infant nutrition. Crit Rev Food Sci Nutr. (2016) 56:1880-92. doi: 10.1080/10408398.2013.803951

28. Zeisel SH, Char D, Sheard NF. Choline, phosphatidylcholine and sphingomyelin in human and bovine milk and infant formulas. J Nutr. (1986) 116:50-8. doi: 10.1093/jn/116.1.50

29. Murase T, Mizuno T, Omachi T, Onizawa K, Komine Y, Kondo H, et al Dietary diacylglycerol suppresses high fat and high sucrose diet-induced body fat accumulation in C57BL/6J mice. J Lipid Res. (2001) 42:372-8.

30. Kamphuis MM, Mela DJ, Westerterp-Plantenga MS. Diacylglycerols affect substrate oxidation and appetite in humans. Am J Clin Nutr. (2003) 77:11339. doi: 10.1093/ajcn/77.5.1133

31. Maceyka M, Spiegel S. Sphingolipid metabolites in inflammatory disease. Nature. (2014) 510:58-67. doi: 10.1038/nature13475

32. Nilsson A. Role of sphingolipids in infant gut health and immunity. J Pediatr. (2016) 173(Suppl.):S53-9. doi: 10.1016/j.jpeds.2016.02.076 
33. Nakagawa Y, Waku K. The metabolism of glycerophospholipid and its regulation in monocytes and macrophages. Prog Lipid Res. (1989) 28:20543. doi: 10.1016/0163-7827(89)90013-1

34. Murray PJ. Immune regulation by monocytes. Semin Immunol. (2018) 35:128. doi: 10.1016/j.smim.2017.12.005

35. Hannun YA, Obeid LM. Sphingolipids and their metabolism in physiology and disease. Nat Rev Mol Cell Biol. (2018) 19:175-91. doi: 10.1038/nrm.2017.107

36. Rollin-Pinheiro R, Singh A, Barreto-Bergter E, Del Poeta M. Sphingolipids as targets for treatment of fungal infections. Future Med Chem. (2016) 8:146984. doi: 10.4155/fmc-2016-0053

37. Astudillo L, Sabourdy F, Therville N, Bode H, Ségui B, Andrieu-Abadie N, et al. Human genetic disorders of sphingolipid biosynthesis. J Inherit Metab Dis. (2015) 38:65-76. doi: 10.1007/s10545-014-9736-1

38. Zhong W, Jeffries MW, Georgopapadakou NH. Inhibition of inositol phosphorylceramide synthase by aureobasidin A in Candida and Aspergillus species. Antimicrob Agents Chemother. (2000) 44:651-3. doi: 10.1128/AAC.44.3.651-653.2000

39. Levery SB, Momany M, Lindsey R, Toledo MS, Shayman JA, Fuller M, et al. Disruption of the glucosylceramide biosynthetic pathway in Aspergillus nidulans and Aspergillus fumigatus by inhibitors of UDP-Glc:ceramide glucosyltransferase strongly affects spore germination, cell cycle, and hyphal growth. FEBS Lett. (2002) 525:59-64. doi: 10.1016/S0014-5793(02)0 3067-3

40. Rittershaus PC, Kechichian TB, Allegood JC, Merrill AH Jr, Hennig M, Luberto C, et al. Glucosylceramide synthase is an essential regulator of pathogenicity of Cryptococcus neoformans. J Clin Invest. (2006) 116:16519. doi: 10.1172/JCI27890

41. Alderson NL, Maldonado EN, Kern MJ, Bhat NR, Hama H. FA2H-dependent fatty acid 2-hydroxylation in postnatal mouse brain. J Lipid Res. (2006) 47:2772-80. doi: 10.1194/jlr.M600362-JLR200

42. Edvardson S, Yi JK, Jalas C, Xu R, Webb BD, Snider J, et al. Deficiency of the alkaline ceramidase ACER3 manifests in early childhood by progressive leukodystrophy. J Med Genet. (2016) 53:389-96. doi: 10.1136/jmedgenet-2015-103457

43. Mosbech MB, Olsen AS, Neess D, Ben-David O, Klitten LL, Larsen J, et al. Reduced ceramide synthase 2 activity causes progressive myoclonic epilepsy. Ann Clin Transl Neurol. (2014) 1:88-98. doi: 10.1002/acn3.28

44. Hirota Y, Nakajima K. Control of neuronal migration and aggregation by reelin signaling in the developing cerebral cortex. Front Cell Dev Biol. (2017) 5:40. doi: 10.3389/fcell.2017.00040

45. Britto JM, Tait KJ, Lee EP, Gamble RS, Hattori M, Tan SS. Exogenous Reelin modifies the migratory behavior of neurons depending on cortical location. Cereb Cortex. (2014) 24:2835-47. doi: 10.1093/cercor/bht123

46. Gärtner A, Fornasiero EF, Munck S, Vennekens K, Seuntjens E, Huttner WB, et al. N-cadherin specifies first asymmetry in developing neurons. Embo J. (2012) 31:1893-903. doi: 10.1038/emboj.2012.41

47. Hong SE, Shugart YY, Huang DT, Shahwan SA, Grant PE, Hourihane JO, et al. Autosomal recessive lissencephaly with cerebellar hypoplasia is associated with human RELN mutations. Nat Genet. (2000) 26:93-6. doi: 10.1038/79246

48. Valledor AF, Hsu LC, Ogawa S, Sawka-Verhelle D, Karin M, Glass CK. Activation of liver $\mathrm{X}$ receptors and retinoid $\mathrm{X}$ receptors prevents bacterialinduced macrophage apoptosis. Proc Natl Acad Sci USA. (2004) 101:1781318. doi: $10.1073 /$ pnas.0407749101

Conflict of Interest: The authors declare that the research was conducted in the absence of any commercial or financial relationships that could be construed as a potential conflict of interest.

Copyright (c) $2020 \mathrm{Xu}$, Chen, Wang, Yu and Han. This is an open-access article distributed under the terms of the Creative Commons Attribution License (CC BY). The use, distribution or reproduction in other forums is permitted, provided the original author(s) and the copyright owner(s) are credited and that the original publication in this journal is cited, in accordance with accepted academic practice. No use, distribution or reproduction is permitted which does not comply with these terms. 